\title{
Double Inlet Left Ventricle with common AV valve, discordant ventriculo-arterial connection and pulmonary atresia: A rare anomaly on fetal echocardiogram with three dimensional spatio-temporal imaging correlation (STIC)
}

\author{
Usha Nandhini Sennaiyan $^{1}$ and Mani Ram Krishna ${ }^{1}$
}

${ }^{1}$ Dr R.K. Hospital for Women and Children

July 13, 2021

\begin{abstract}
Double Inlet Left Ventricle through a common AV valve is a rare type of functionally univentricular heart. We report a fetus with double inlet left ventricle with discordant ventriculo-arterial connection and pulmonary atresia. The anatomy was readily apparent on three dimensional rendering by spatio-temporal imaging correlation technique(STIC)
\end{abstract}

Double Inlet Left Ventricle with common AV valve, discordant ventriculo-arterial connection and pulmonary atresia: A rare anomaly on fetal echocardiogram with three dimensional spatiotemporal imaging correlation (STIC)

Usha Nandhini Sennaiyan and Mani Ram Krishna

Dr R.K. Hospital for Women and Children

Thanjavur, Tamil Nadu, India

Correspondence to

Dr Mani Ram Krishna

Dr R.K. Hospital for Women and Children,

No 7, V.O.C.Nagar, Thanjavur - 613007.

Tamil Nadu, India.

E-mail: mann_comp@hotmail.com

Conflict of Interest: The authors report no conflict of interest with regard to this work.

Funding: There was no external funding involved in the submitted work

Abstract:

Double Inlet Left Ventricle through a common AV valve is a rare type of functionally univentricular heart. We report a fetus with double inlet left ventricle with discordant ventriculo-arterial connection and pulmonary atresia. The anatomy was readily apparent on three dimensional rendering by spatio-temporal imaging correlation technique (STIC) 
A pregnant woman was referred to us at 21 weeks gestational age for evaluation of a suspected congenital heart disease. On fetal echocardiogram, there was usual arrangement of the abdominal organs and the atria. The heart was in the left side of the chest with the apex pointing to the left. The systemic and pulmonary veins drained normally. There was a large primary type of atrial septal defect (Fig 1A). There was univentricular atrio-ventricular $(\mathrm{AV})$ connection to a dominant left ventricle through a common $\mathrm{AV}$ valve (Fig $1 \mathrm{~A}$ and Video 1). A small incomplete ventricle was imaged anterior and to the left of the dominant ventricle (Fig 1 $\mathrm{B}$ and Video 2). The aorta arose from the incomplete ventricle (Fig 1D) and was unobstructed. There was pulmonary atresia with retrograde flow in the arterial duct to the pulmonary trunk (Fig 1D and Video 3). The anatomy was readily apparent on three dimensional rendering by spatio-temporal imaging correlation (STIC) technique ( Fig 1B and Videos 1 to 3)

Double Inlet Left Ventricle is the commonest type of functionally univentricular heart with univentricular AV connection (1). The most common types of DILV have discordant ventriculo-arterial connections (2). A very small proportion of DILV have a primary type of atrial septal defect with the AV junction guided by a common AV valve (3). To the best of our knowledge, this anatomy has not been reported previously during a fetal echocardiogram.

Figure Legends:

Figure 1A: A fetal echocardiogram image in the 4-chamber view demonstrating the inter-atrial septum and a large primary type atrial septal defect (green star). The atria are connected to a single dominant ventricle through a common $\mathrm{AV}$ valve

Figure 1B: An image from a three-dimensional rendering of the fetal heart by spatio-temporal imaging correlation (STIC) demonstrating the incomplete ventricle which is present anterior and to the left of the dominant ventricle

Figure 1C: A fetal echocardiogram image demonstrating an unobstructed aortic outflow arising from the incomplete ventricle (RV)

Figure 1D: A fetal echocardiogram image from the three-vessel tracheal view demonstrating reversal of flow (red color) in a vertically aligned arterial duct

Video 1: A three-dimensional fetal echocardiogram loop rendered by spatio-temporal imaging correlation (STIC) demonstrating the common AV valve when viewed from the ventricular aspect

Video 2: A three-dimensional fetal echocardiogram loop rendered by spatio-temporal imaging correlation (STIC) demonstrating the dominant left ventricle and the incomplete ventricle from which the aorta arises

Video 3: A three-dimensional fetal echocardiogram loop with color Doppler rendered by spatio-temporal imaging correlation (STIC) demonstrating forward flow in the aorta (blue) and reversal of flow in the arterial duct(red)

References

1. Van Praagh R, Ongley PA, Swan HJ. Anatomic types of single or common ventricle in man: morphologic and geometric aspects of 60 necropsied cases. Am J Cardiol. 1964; 13 : 367-386

2. Khairy P, Poirier N and Mercier L. Univentricular Heart.Circulation. 2007; 115: 800-812

3. Al-Ethawi AESD and Al-Kaaby BA. The univentricular heart: Revisited.Cir Cardiov. 2018; 25(3): 141-147. 

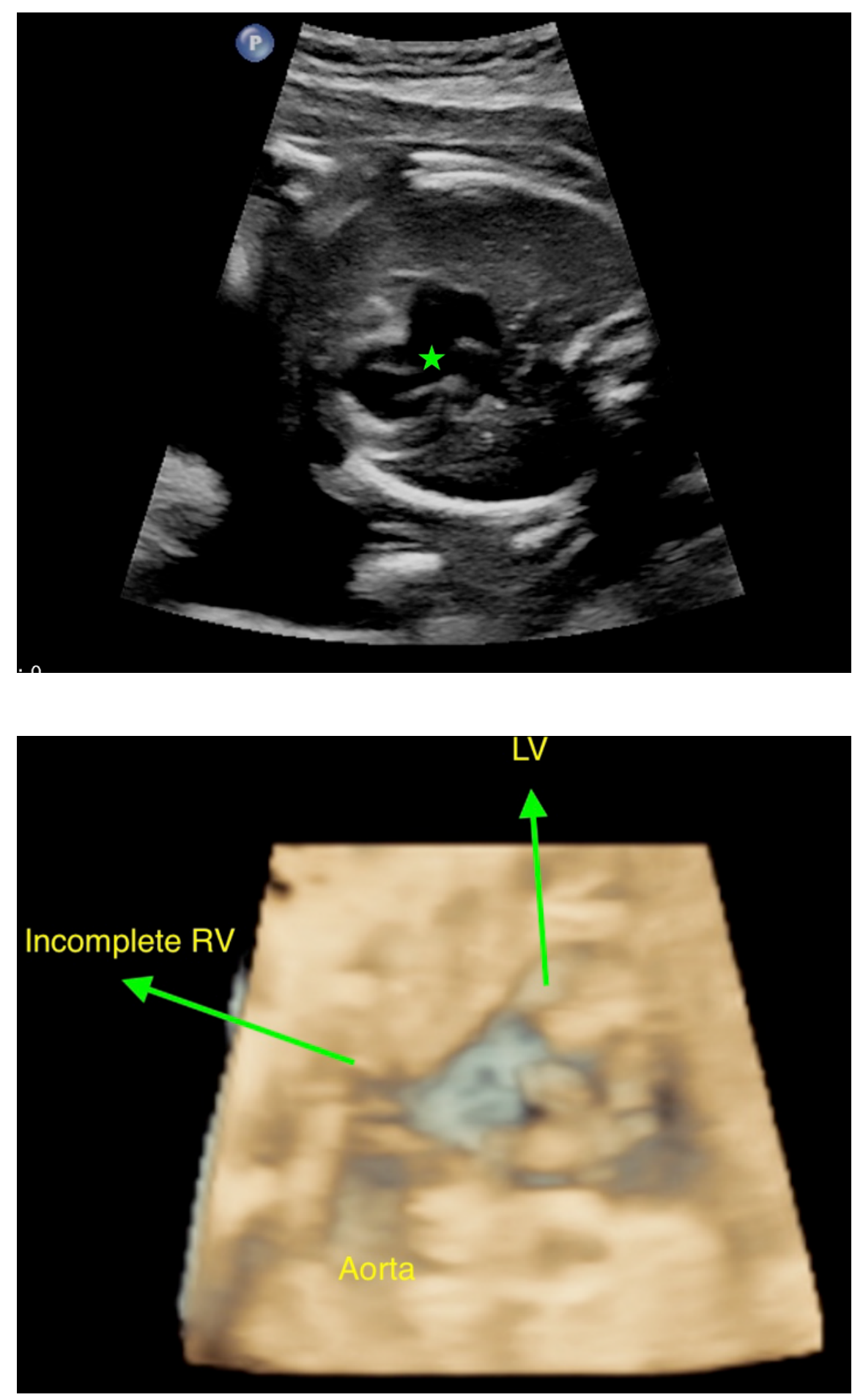

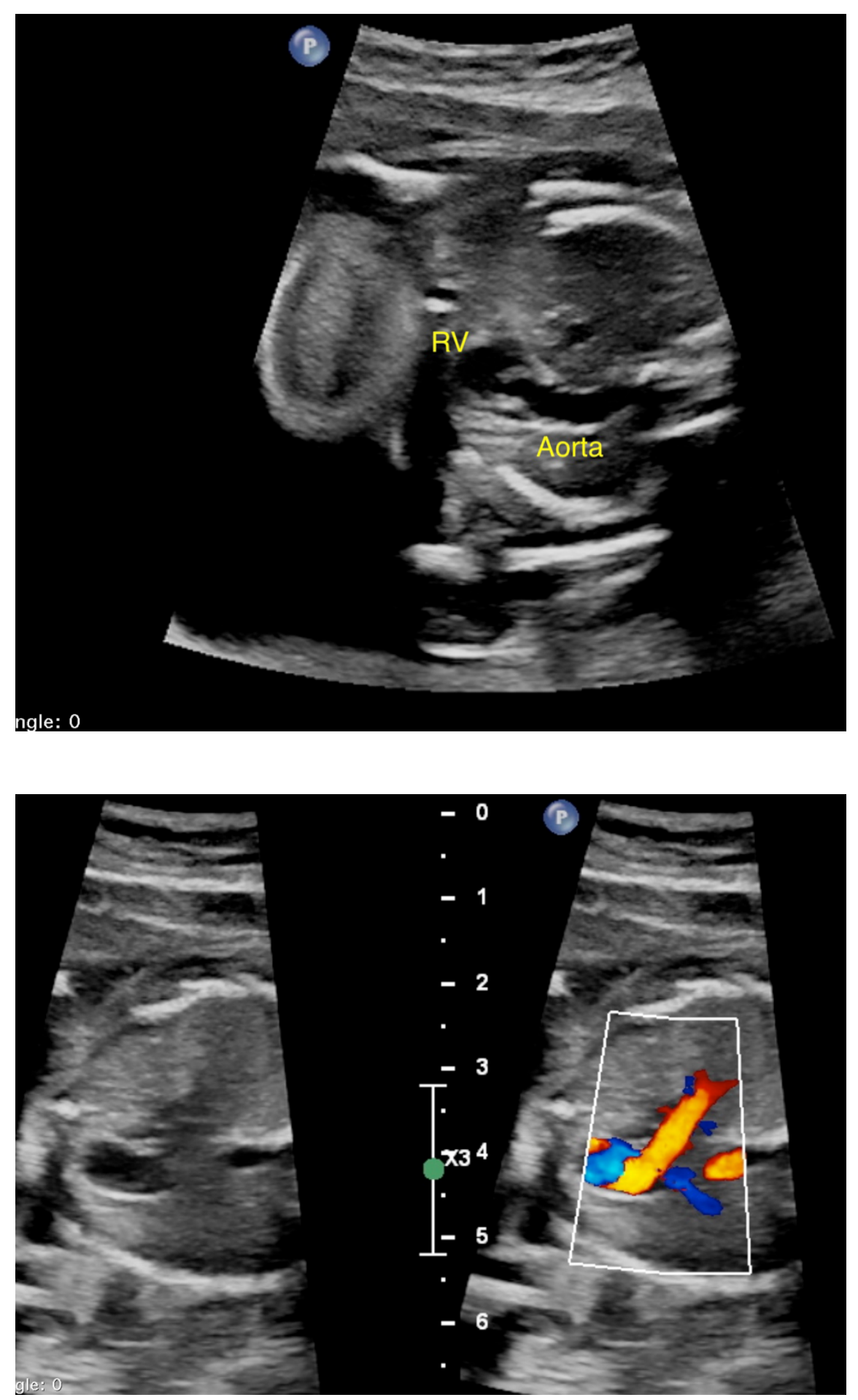